\title{
Berberine Upregulates P-Glycoprotein in Human Caco-2 Cells and in an Experimental Model of Colitis in the Rat via Activation of Nrf2-Dependent Mechanisms $\underline{\underline{S}}$
}

\author{
Wanghui Jing, Yasaman Safarpour, Ting Zhang, Pengqi Guo, Guoning Chen, Xiaoming Wu, \\ Qiang Fu, and Yitao Wang
}

School of Pharmacy (W.J., T.Z., P.G., G.C., Q.F.) and School of Life Science and Technology (X.W.), Xi'an Jiaotong University, Xi'an, PR China; School of Medicine, University of California, Irvine, California, (W.J., Y.S.); and State Key Laboratory of Quality Research in Chinese Medicine, Institute of Chinese Medical Sciences, University of Macau, Macau, China (P.G., Y.W.)

Received April 3, 2018; accepted June 6, 2018

\begin{abstract}
Downregulation of $\mathrm{P}$-glycoprotein $(\mathrm{P}-\mathrm{gp})$ is implicated in the pathophysiology of inflammatory bowel disease (IBD). Berberine, a principal isoquinoline alkaloid extracted from Berberis species, has been reported to exhibit therapeutic potential in IBD. In this study, we used a dextran sulfate sodium (DSS)-induced colitis rat model to evaluate the effect of berberine on P-gp and explore its mechanism of action. Berberine treatment improved DSS-induced colitis symptoms, attenuated inflammatory markers (myeloperoxidase, tumor necrosis factor- $\alpha$, and interleukin- $1 \beta$ and -6 ), and enhanced P-gp expression in a dose-dependent manner. Although colonic expression of the $\mathrm{P}$-gp-related nuclear receptor pregnane $\mathrm{X}$ receptor and transcription factor nuclear factor erythroid 2-related factor 2 (Nrf2) were downregulated in the colitis model, gene and protein expression analysis revealed
\end{abstract}

\section{Introduction}

Inflammatory bowel disease (IBD) is a chronic inflammatory disorder that affects the gastrointestinal tract, and its incidence and clinical severity have grown worldwide. IBD comprises two different diseases: ulcerative colitis (UC) and Crohn disease. Symptoms include diarrhea, abdominal pain, body weight loss, rectal bleeding, malnutrition, and fever (Zhang et al., 2015a). Currently, the exact causes of IBD are poorly understood; however, impaired barrier function of the gut epithelium is reported to be an important factor in IBD pathogenesis (MinerWilliams and Moughan, 2016; Tili et al., 2017).

This work was supported financially by the National Natural Science Foundation of China [Grant 81603370], Natural Science Foundation of Shaanxi Province [Grant 2017JQ8006], the Fundamental Research Funds for the Central Universities [Grant xjj2016085], International Postdoctoral Exchange Fellowship Program [Grant 20150050], and Shanxi Postdoctoral Science Foundation.

https://doi.org/10.1124/jpet.118.249615.

S This article has supplemental material available at jpet.aspetjournals.org. that berberine treatment reversed only the downregulation of Nrf2. In vitro studies using Caco-2 cells showed that the multidrug resistance 1 (MDR1) gene and P-gp protein were upregulated by berberine in a dose- and time-dependent manner. Significant upregulation of the MDR1 gene by berberine was abrogated by Nrf2 silencing, indicating that the Nrf2-mediated pathway was responsible for this activation. Luciferase assays showed a dose-dependent increase in Nrf2 reporter gene activity after berberine treatment in Caco-2 cells, with a significant 2-fold elevation at $2.5 \mu \mathrm{M}$ berberine, suggesting that berberine is a strong Nrf2 activator. These results indicate the possible involvement of Nrf2-mediated upregulation of $\mathrm{P}$-gp in the therapeutic effect of berberine on colitis and highlight the potential of P-gp and/or Nrf2 as new therapeutic targets for IBD.

ABBREVIATIONS: BBB, berberine; DAI, disease activity index; DSS, dextran sulfate sodium; ELISA, enzyme-linked immunosorbent assay; GAPDH, glyceraldehyde 3-phosphate dehydrogenase; IBD, inflammatory bowel disease; IL, interleukin; Keap1, Kelch-like ECH-associated protein 1; MDR1, multidrug resistance 1; MPO, myeloperoxidase; Nrf2, nuclear factor erythroid 2-related factor 2; P-gp, P-glycoprotein; PXR, pregnane X receptor; qRT-PCR, quantitative real-time polymerase chain reaction; rho123, rhodamine 123; SFN, sulforaphane; siRNA, small interfering RNA; TNF, tumor necrosis factor; UC, ulcerative colitis. 
(Chu et al., 2006). In addition, several studies characterizing the association between $M D R 1$ polymorphisms and IBD susceptibility (Brinar et al., 2013; Zhao et al., 2015) have indicated that stimulating P-gp expression and function could be a new treatment of IBD.

The nuclear receptor pregnane $\mathrm{X}$ receptor (PXR) plays a major role in activating P-gp expression (Sehirli et al., 2015). Although PXR dysregulation in the intestine likely contributes to UC pathophysiology (Cheng et al., 2010), PXR activation has been demonstrated to be an effective treatment in an experimental colitis mouse model and in human cell lines (Zhang et al., 2015a,b). Nuclear factor erythroid 2-related factor 2 (Nrf2), a cytoprotective transcription factor against oxidative stress, plays an important role in the antioxidant response by regulating the transcription of several detoxifying/antioxidant enzymes and transporters, including P-gp (Aleksunes and Klaassen, 2012). Nrf2 ${ }^{-/-}$mice exhibit increased sensitivity to dextran sulfate sodium (DSS)mediated colitis (Khor et al., 2006), and activation of Nrf2 by its inducer sulforaphane (SFN) increases P-gp expression and function at the rat blood-brain barrier (Wang et al., 2014). These reports suggest that Nrf2 may mediate P-gp dysregulation in the intestinal barrier in colitis.

Berberine is the major isoquinoline alkaloid in the stems and roots of Berberis species. Several studies have reported the therapeutic potential of berberine for IBD (Hong et al., 2012; Yan et al., 2012; Li et al., 2016; Liu et al., 2018). Among the numerous possible mechanisms involved in the efficacy of berberine in IBD (Habtemariam, 2016), recent reports have focused on berberine-mediated improvements in gut epithelial barrier dysfunction and the role of tight junction proteins zona occludens-1 and occludin (Gu et al., 2009; Yan et al., 2012; Tan et al., 2015; Li et al., 2016). Numerous reports have demonstrated that berberine is a P-gp substrate and that it regulates P-gp expression and function (Lin et al., 1999b; Shitan et al., 2007; Qiu et al., 2009; Zhang et al., 2011; Shan et al., 2013). Most of these studies were performed in vitro, however, and some were also controversial. Whether berberine improves the intestinal barrier by regulating P-gp expression in a DSSinduced colitis rat model has yet to be determined. Another question is whether and how the colitis-related nuclear receptor PXR and nuclear factor Nrf2 contribute to gut barrier healing if berberine exhibits a regulatory effect on P-gp.

Therefore, in the current study, the effect of berberine on P-gp expression in the colon was assessed in a DSS-induced colitis rat model. For further mechanistic investigation, the regulation of $\mathrm{P}$-gp expression and activity in Caco-2 cells, a human epithelial colorectal adenocarcinoma cell line that naturally expresses the $M D R 1$ gene, in response to berberine treatment were also characterized.

\section{Materials and Methods}

Chemicals and Reagents. Berberine hydrochloride was obtained from Shanghai Boyun Biotech Co. (Shanghai, China) at the highest available purity ( $\geq 95 \%$ ). DSS (mol. wt. 36,000-50,000) was purchased from MP Biomedicals (Santa Ana, CA). Nrf2 small interfering RNA (siRNA) and control siRNA were obtained from Invitrogen Life Technologies (Shanghai, China). Dulbecco's modified Eagle's medium, fetal bovine serum, streptomycin, Triton $\mathrm{X}-100$, and TRIzol reagent were purchased from Sigma-Aldrich (St. Louis, MO).
Animal Care and Experimental Design. Male Sprague-Dawley rats $(\sim 250 \mathrm{~g})$ were obtained from the Experimental Animal Center of Xi'an Jiaotong University (Xi'an, China) [license no. SCXK (Shaanxi) 2012-003]. Animals were housed in a climate-controlled vivarium with a relative humidity of $55 \% \pm 5 \%$ under 12 -hour day/night cycles and provided with food and water ad libitum.

Rats were divided into four groups: normal, colitis, colitis + $10 \mathrm{mg} / \mathrm{kg}$ berberine (BBR LD), and colitis $+40 \mathrm{mg} / \mathrm{kg}$ berberine (BBR HD). Colitis was induced by administration of 5\% (w/v) DSS in drinking water for 7 days. Berberine was dissolved in water and administered to the BBR $\mathrm{LD}$ and $\mathrm{BBR}$ HD groups via oral gavage during those 7 days. In parallel, the same quantity of water was administered to the rats from the control and colitis groups via oral gavage for 7 days. The berberine treatment dosage was determined based on a previous report ( $\mathrm{Li}$ et al., 2016), and a preliminary experimental result in our laboratory (data not shown). This study was approved by the Ethical Committee of Xi'an Jiaotong University, and studies were performed in accordance with the Guide for the Care and Use of Laboratory Animals of the Ethical Committee of Xi'an Jiaotong University, Xi'an, China (permit no. XJTU 2011-0045).

Daily Observation and Sample Collection. Rats were monitored daily for stool consistency, body weight, and rectal bleeding during the experimental period. Disease activity index (DAI) was measured as described previously in our laboratory (Huang et al., 2015). On the final day, animals were sacrificed under anesthesia, and the colon was immediately obtained to identify ulcers in the colonic mucosa and measure colon length and weight. Additionally, $1 \mathrm{~cm}$ of the distal colon was excised for histologic evaluation. The remaining colon was stored in liquid nitrogen for quantitative real-time polymerase chain reaction ( $q R T-P C R)$, enzyme-linked immunosorbent assay (ELISA), and Western blot analysis. The spleen was removed to measure its weight, as spleen weight represents an index of systemic inflammation (Antonioli et al., 2007).

Histologic Analysis of Colon Tissues. The colon tissues were removed and fixed with $10 \%$ formalin solution overnight. Histologic sections were stained with H\&E. Colon injury and inflammation were graded on a $0-5$ scale, as described in previous reports (Maeda et al., 2005; Jing et al., 2016).

Evaluation of Myeloperoxidase Activity in Colonic Mucosa. Myeloperoxidase (MPO) activity is used to measure the degree of inflammation in colon tissue (Metzler et al., 2011). Its activity in the colonic mucosa was evaluated as described previously (Huang et al., 2015). One unit of MPO activity indicates the quantity of enzyme required for converting $1 \mathrm{nmol}$ of hydrogen peroxide to water per minute at $\sim 25^{\circ} \mathrm{C}$.

Determination of Colonic Cytokines. The levels of IL- $1 \beta$, IL-6, and tumor necrosis factor (TNF- $\alpha$ ) in colon homogenates were determined with ELISA (R\&D Systems, Minneapolis, MN) according to the protocol of the manufacturer.

Cell Culture. Caco-2 cells (American Type Culture Collection, Rockville, MD) were cultured in Dulbecco's modified Eagle's medium containing $10 \%$ fetal bovine serum, $100 \mathrm{U} / \mathrm{ml}$ penicillin, and $100 \mu \mathrm{g} / \mathrm{ml}$ streptomycin. The cell medium was changed with new medium every 2 days. Cells were passaged upon reaching $\sim 80 \%$ confluence.

Drug or siRNA Treatment in Caco-2 Cells. Caco-2 cells were plated in six-well plates at a density of $8 \times 10^{5}$ cells/well and incubated at $37^{\circ} \mathrm{C}$. After 24 hours, the cells were treated with $0,0.1,0.5$, and 2.5 $\mu \mathrm{M}$ berberine for 48 hours or with $2.5 \mu \mathrm{M}$ berberine for $0,12,24$, or 48 hours. For RNA silencing, we used HiPerFect transfection reagent (Qiagen, Hilden, Germany) to transfect Caco-2 cells with $100 \mathrm{nM}$ siRNA targeting human Nrf2 (Hs_NRF2L2_7) or negative control siRNA (Allstar Negative Control siRNA; Qiagen), followed by incubation for 72 hours. During the final 48 hours, $2.5 \mu \mathrm{M}$ berberine was added to the culture medium. Cells were then rinsed, scraped, and used for Western blotting or qRT-PCR analysis.

Uptake of Rhodamine 123 in Caco-2 Cells. Uptake of rhodamine 123 (rho123) in Caco-2 cells was measured for the evaluation of P-gp activity (Zhang et al., 2011). After pretreatment with berberine at 
$0,0.1,0.5$, or $2.5 \mu \mathrm{M}$ for 48 hours or with $2.5 \mu \mathrm{M}$ berberine for $0,12,24$, or 48 hours, Caco-2 cells were washed with Hank's balanced salt solution (Biochrom, Berlin, Germany) and treated with $5 \mu \mathrm{M}$ rho123 for 120 minutes. At the end of the experiment, cells were washed again and lysed with Triton X-100. Cell lysate $(150 \mathrm{ml})$ was transferred to a black 96-well microplate (BD Biosciences, Franklin Lakes, NJ), and the fluorescence intensity of rho123 was determined (excitation wavelength: $485 \mathrm{~nm}$; emission wavelength: $538 \mathrm{~nm}$ ) using a WALLAC multilabel/luminescence counter (PerkinElmer, Waltham, MA).

qRT-PCR. Total RNA was extracted from colon tissues and Caco-2 cells using TRIzol reagent according to the protocols of the manufacturer. RNA concentrations were calculated by spectroscope at $260 \mathrm{~nm}$. Total RNA ( $5 \mu \mathrm{g})$ was reverse-transcribed into single-stranded cDNA using the Superscript III First-Strand Synthesis System (Invitrogen, Carlsbad, CA) for RT-PCR. The cDNA samples were subjected to qPCR with SYBR Green in an ABI 7500 RT-PCR system (Applied Biosystems, Foster City, CA). The primers for mRNA quantification are shown in Supplemental Table 1. Reactions involved the following protocol: $95^{\circ} \mathrm{C}$ for 5 minutes, followed by 40 cycles of $95^{\circ} \mathrm{C}$ for 15 seconds and $60^{\circ} \mathrm{C}$ for 1 minute. The mRNA levels were calculated by the $\triangle \triangle \mathrm{C}_{\mathrm{T}}$ method, and data were normalized to levels of glyceraldehyde 3-phosphate dehydrogenase (GAPDH).

Western Blotting Analysis. Cytoplasmic and nuclear extracts of colon tissues and cells were prepared as described in our previous reports (Jing et al., 2016). Proteins of interest in the cytoplasmic and nuclear fractions of colon tissues or cells were measured by Western blotting as described in our previous report (Jing et al., 2016, 2017) using the following antibodies: anti P-gp (1:400, cat. no. 517310; Calbiochem, San Diego, CA), anti-PXR (1:1000, cat. no. ab118336; Abcam, Cambridge, MA), anti- Kelch-like ECH-associated protein 1 (Keap1; 1:1000, cat. no. ab139729; Abcam), and anti-Nrf2 (1:1000, cat. no. ab89443; Abcam). Mouse antibodies against histone H3 (1:10,000, cat. no. ab32356; Abcam) and GAPDH (1:10,000, cat. no. ab8245; Abcam) were used to measure nuclear and cytosolic housekeeping proteins, respectively.

Luciferase Assay. Caco-2 cells were plated in 96-well plates at a density of $5 \times 10^{4}$ cells/well and incubated at $37^{\circ} \mathrm{C}$. After 24 hours, the cells were transfected with pGL3-ARE-Luc, pEF-Nrf2, and pRL-TK (Promega, Madison, WI) using LipofectAMINE 2000 (Invitrogen) according to the protocols of the manufacturer. After transfection, Caco-2 cells were treated with SFN $(10 \mu \mathrm{M})$ or berberine $(0.1,0.5$, or $2.5 \mu \mathrm{M})$ for 24 hours, and then cell lysates were prepared. Luciferase activity was measured using a SpectraMax M5 multi-mode microplate reader (Molecular Devices, San Jose, CA). Firefly luciferase activity was normalized to that of Renilla luciferase, and activity was expressed as fold induction relative to blank control.

Data Analysis. Data are presented as the mean \pm S.E.M. All statistical evaluations were performed using GraphPad Prism 6.0 software (GraphPad, San Diego, CA) with unpaired Student's $t$ test or one-way analysis of variance. A value of $P<0.05$ was considered statistically significant.

\section{Results}

Berberine Alleviated DSS-Induced Colitis. A colitis rat model was successfully developed using $5 \%$ DSS, resulting in severe colitis-like symptoms. The DAI, including stool consistency, body weight loss, and gross bleeding, was elevated after DSS administration for 2 days (Fig. 1A), in agreement with our previous study (Jing et al., 2016). Rats with DSS-induced colitis treated with berberine (10 and $40 \mathrm{mg} / \mathrm{kg}$ ) for 7 days showed reduced responses to DSS and lowering of DAI scores (Fig. 1A). An increase in the weightlength ratio of the colon in a colitis rat model is a major biologic marker for colonic inflammation (Martin et al., 2016). In the current study, rats with DSS-induced colitis showed an increased colon weight-length ratio after DSS administration for 1 week, whereas berberine treatment (10 and $40 \mathrm{mg} / \mathrm{kg}$ ) reversed this trend (Fig. 1D). Splenomegaly is common among patients with UC (Ashrafi et al., 2014), and spleen weight represents an index of systemic inflammation (Antonioli et al., 2007). In agreement with earlier studies (Jing et al., 2016), enlarged spleens were observed in DSS-treated rats, and this was significantly attenuated by berberine $(10$ and $40 \mathrm{mg} / \mathrm{kg})$ treatment (Fig. 1, B and C). Inflammation of the colonic mucosa in UC is characterized mainly by neutrophil accumulation, which is quantified by measuring MPO activity. We observed increased MPO activity in the colonic mucosa from DSS-treated rats, and we found that berberine administration significantly attenuated this DSS-triggered MPO activation (Fig. 1E). Additionally, histologic studies of the distal colon tissues demonstrated that the severity and extent of inflammatory lesions in the colons of DSS-treated rats were significantly greater than those observed in normal rats; moreover, treatment with $40 \mathrm{mg} / \mathrm{kg}$ berberine markedly reduced the histology scores in the colitis group (Supplemental Fig. S1).

Berberine Relieved DSS-Induced Inflammation in the Colon. After DSS administration for 7 days, we measured the concentrations of IL- $1 \beta$, IL- 6 , and TNF- $\alpha$ in colon tissue. The results shown in Fig. 2 indicate that levels of these pro-inflammatory cytokines were much higher in DSS-treated rats than in the normal group. Increases in inflammatory cytokine levels in the DSS-treated rats were significantly attenuated by berberine administration.

Berberine Altered P-gp and Nrf2 Expression in Colons from Rats With DSS-Induced Colitis. The colonic gene expression of $m d r 1 a$ was analyzed by qRT-PCR. Figure 3 shows that DSS administration markedly decreased the expression of mdr1a mRNA. In comparison, berberine treatment reversed this reduction in mdr1a in the DSS-treated group, inducing levels that were higher than normal. To characterize further the mechanism of action of berberine on mdr1a expression, we determined the mRNA expression of nuclear receptor $P X R$ and transcription factor $N r f 2$, which are related to colitis. As shown in Fig. 3, both PXR and Nrf2 mRNA levels were significantly decreased in response to DSS administration. Berberine treatment, however, restored only the Nrf2 mRNA level and had no effect on PXR mRNA expression. These results indicate that $N r f 2$, but not PXR, may play a critical role in berberine-mediated P-gp induction in the DSS-induced colitis model.

To confirm the preceding results regarding berberineinduced upregulation of $m d r 1 a$ and $N r f 2$ gene expression, we evaluated the protein expression of P-gp, PXR, and Nrf2 using Western blotting. Compared with those in normal rats, the protein expression of P-gp, PXR, and nuclear Nrf2 was decreased in DSS-treated rats. Berberine administration significantly reversed the downregulation of P-gp and nuclear Nrf2 in the DSS-treated group (Fig. 4); however, consistent with the results of the mRNA expression experiment, berberine exhibited no effect on PXR protein expression. Taken together, our results suggest that berberine upregulates the mRNA and protein levels of P-gp, possibly via Nrf2 activation.

Berberine Upregulated P-gp in a Dose- and TimeDependent Manner. To characterize the mechanism of P-gp upregulation by berberine in vitro, Caco-2 cells were incubated with berberine $(0,0.1,0.5$, or $2.5 \mu \mathrm{M})$ for 48 hours. Results in Fig. 5, A and B show that the expression of $M D R 1$ gene and P-gp protein was significantly upregulated by berberine treatment 
A

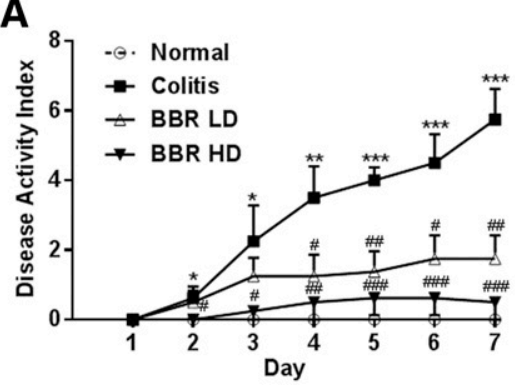

B

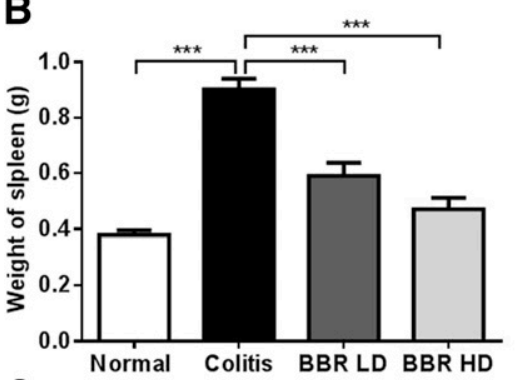

C

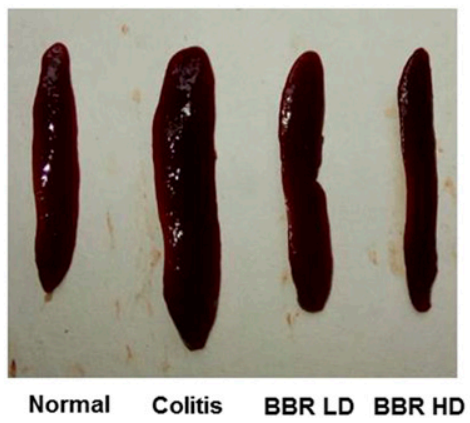

D

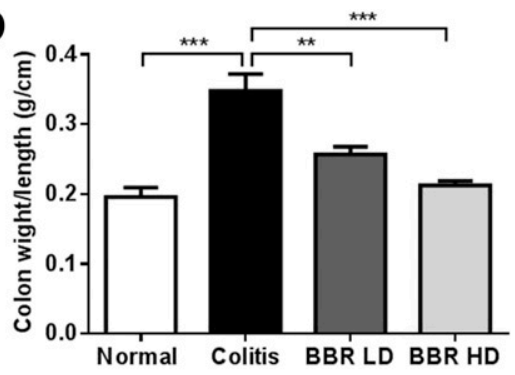

E

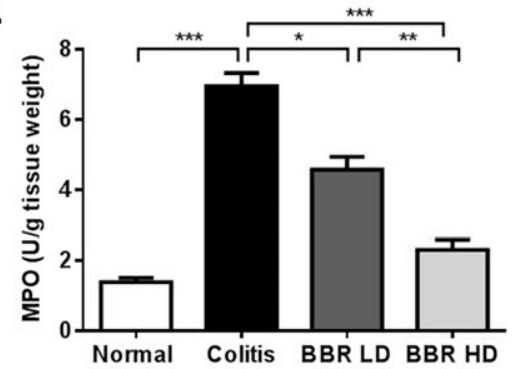

Fig. 1. Berberine improved colitis-like symptoms in DSS-induced colitis rats. (A) DAI, (B and C) spleen weight, (D) colon weight-length ratio, and (E) MPO activity in colon. Data represent the mean \pm S.E.M. $(n=$ 8 rats per group). ${ }^{*} P<0.05 ; * * P<0.01 ; * * * P<0.001$. $\mathrm{BBR}$, berberine; $\mathrm{HD}$, high dose; $\mathrm{LD}$, low dose. at concentrations of 0.5 and $2.5 \mu \mathrm{M}$. P-gp function was evaluated by assessing the uptake of rho123, a P-gp substrate. After berberine treatment $(0.1,0.5$, or $2.5 \mu \mathrm{M})$, intracellular uptake of rho123 was significantly decreased (Fig. 5C), indicating an increase in P-gp function in response to berberine treatment. Furthermore, we evaluated P-gp regulation in Caco2 cells by incubating them with $2.5 \mu \mathrm{M}$ berberine for $0,12,24$, or 48 hours. Results showed that, in addition to inducing dosedependent effects, berberine treatment increased MDR1 gene expression and P-gp protein expression and function in a timedependent manner (Fig. 5, D-F).

Berberine Induced P-gp Expression and Function Via Nrf2 in Caco-2 Cells. To investigate further whether activation of Nrf2 is necessary for berberine-mediated P-gp upregulation, an Nrf2 gene-silencing experiment was conducted in Caco-2 cells. The results in Fig. 6A show that Nrf2 expression was decreased by $\sim 70 \%$ after transfection with $N r f 2$-siRNA. qRTPCR was used to analyze changes in MDR1 gene expression. Figure $6 \mathrm{~B}$ shows that $N r f 2$ silencing significantly reduced $M D R 1$ mRNA expression to $54 \%$ that in the negative control; moreover, activation of $M D R 1$ by berberine was abolished by Nrf2 silencing (Fig. 6B). These results demonstrate that berberine induces MDR1 gene expression via Nrf2 activation in Caco-2 cells.

Effects of Berberine on Nrf2 Activation in Caco-2 Cells. Although the effect of berberine on the mRNA expression of $N r f 2$ was investigated in vivo, it may not accurately represent the effect of berberine on $N r f 2$ gene function. Therefore, the effect of berberine on Nrf2 activation was determined using a luciferase reporter assay in Caco-2 cells, which were transiently transfected with reporter plasmids. As shown in Fig. 6C, Nrf2 reporter gene activity in the SFN group increased significantly to a level 2.5-fold of that in the control group; similarly, berberine increased Nrf2 reporter gene activity in a dose-dependent manner, with a significant 2 -fold elevation at $2.5 \mu \mathrm{M}$ berberine. To confirm the regulatory effect of berberine on the nuclear translocation of $\mathrm{Nrf} 2$, we also measured the abundance of the Nrf2 inhibitor Keap1 in the cytoplasm and Nrf2 expression in the nucleus of Caco- 2 cells in response to berberine $(2.5 \mu \mathrm{M})$ and SFN treatment. Treatment with the Nrf2 activator SFN significantly reduced cytoplasmic Keap1 abundance and significantly increased the nuclear translocation of Nrf2; similar results were observed after treatment with berberine (Fig. 6, D and E). These data demonstrate the role of berberine as a strong Nrf2 activator.

\section{Discussion}

Rats with DSS-induced colitis showed symptoms similar to those associated with UC patients, including diarrhea, body weight loss, bloody stool, and mucosal ulceration, and this model is used extensively for basic research and drug 

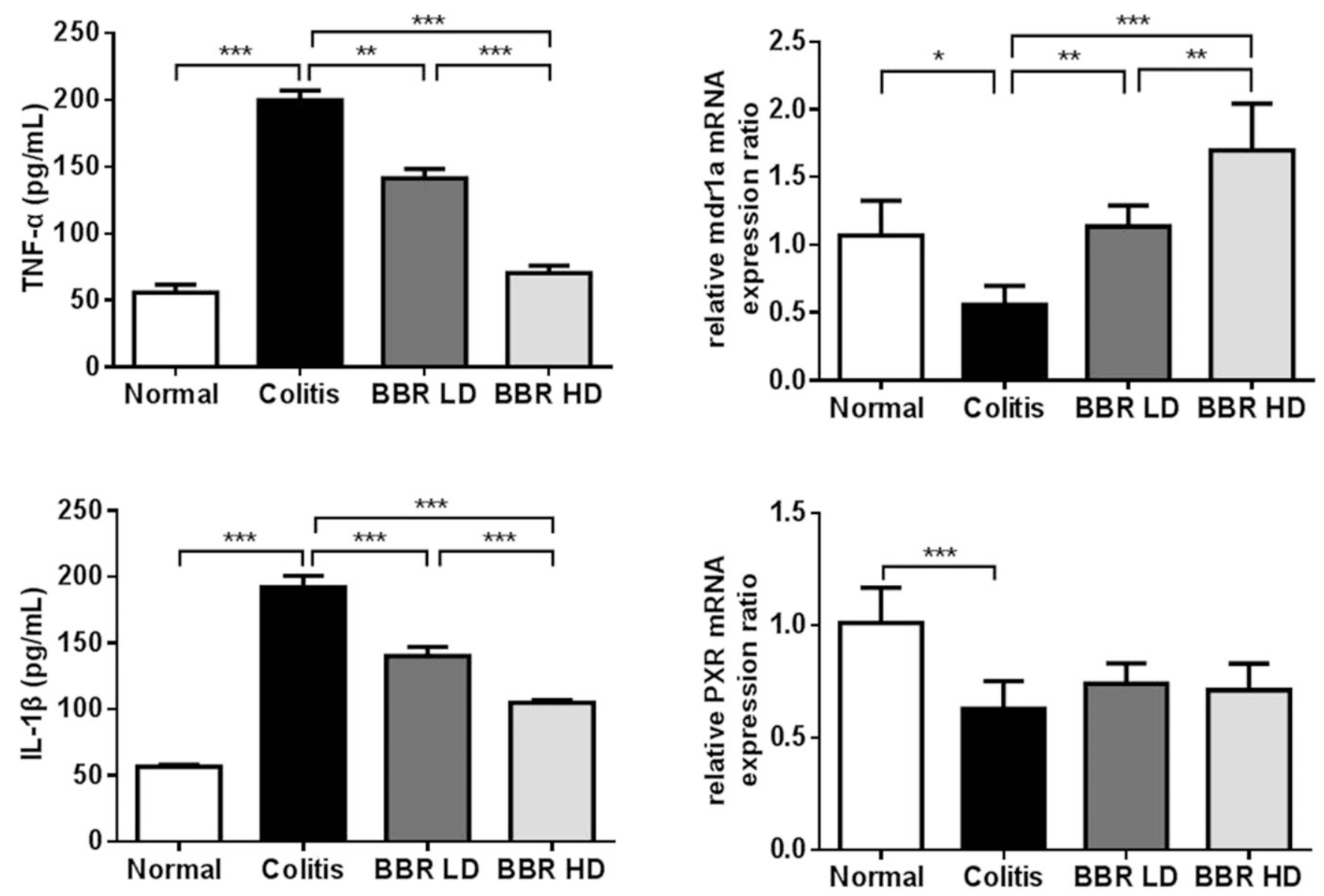

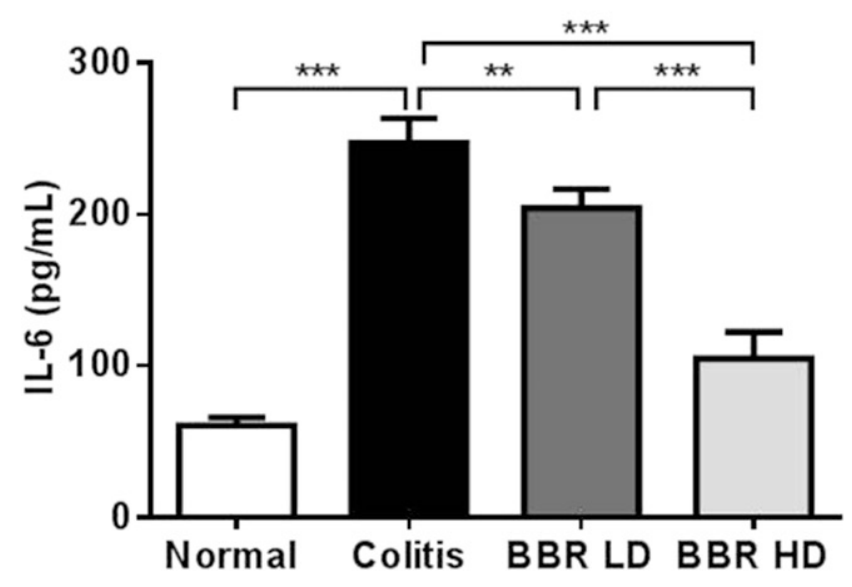

Fig. 2. Berberine relieved DSS-induced inflammation in colon tissues. Protein concentrations of the inflammation-related cytokines TNF- $\alpha$, IL-1 $\beta$, and IL- 6 in colonic homogenates were determined by ELISA. Data represent the mean \pm S.E.M. $\left(n=8\right.$ rats per group). ${ }^{* *} P<0.01$; $* * * P<$ 0.001 . HD, high dose; LD, low dose.

discovery. In this study, using the DSS-induced colitis model, we observed neutrophil infiltration in colon tissues, as evidenced by increased MPO activity in the colonic mucosa, as well as splenomegaly, which is commonly reported in human UC (Ashrafi et al., 2014; Peterson et al., 2016) (Fig. 1). After DSS administration, we also observed increased levels of proinflammatory cytokines in colon tissues (TNF- $\alpha$, IL- $1 \beta$, and IL-6). These findings are similar to those of our previous study

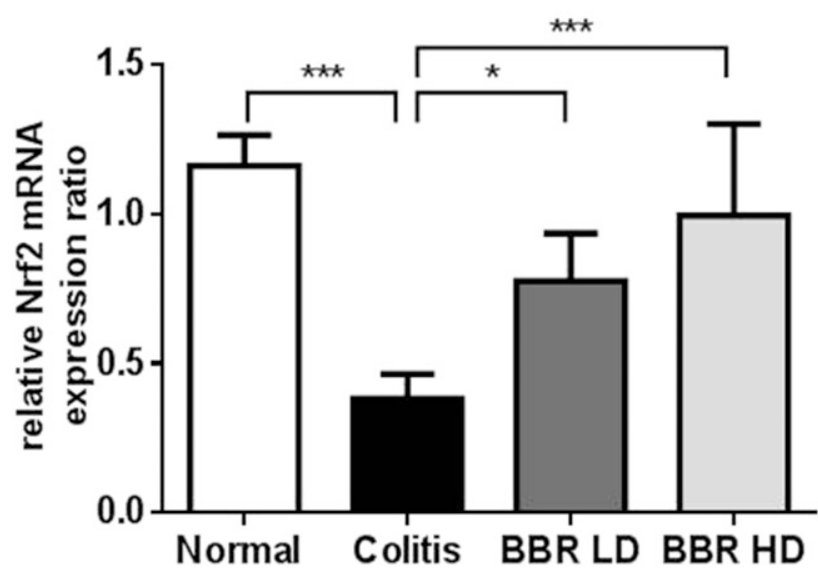

Fig. 3. Berberine regulated the expression of $m d r 1 a$ and $N r f 2$ and had no effect on PXR expression in DSS-induced colitis rats. qRT-PCR was performed to measure the gene expression of $m d r 1 a, P X R$, and Nrf2. Data represent the mean \pm S.E.M. $\left(n=8\right.$ rats per group). ${ }^{*} P<0.0$; ${ }^{* *} P<0.01$; $* * * P<0.001$. HD, high dose; LD, low dose.

involving rats with colitis induced by $5 \%$ DSS administration (Jing et al., 2016). In this study, berberine treatment alleviated colitis symptoms in a dose-dependent manner (Figs. 1 and 2). Although similar results have been reported previously (Zhou and Mineshita, 2000; Lee et al., 2010; Hong et al., 2012; Yan et al., 2012; Li et al., 2016), these studies used different colitis models and different rodents. This is the first report showing an improvement in DSS-induced colitis 


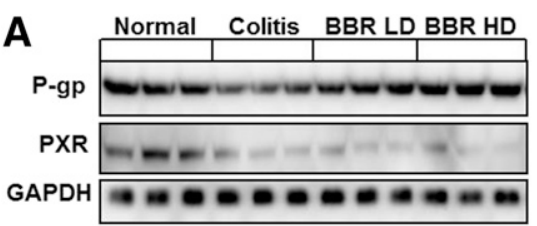

B

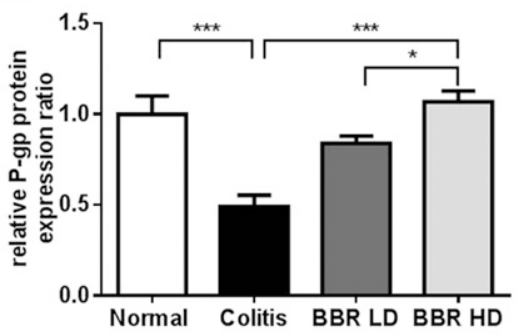

C

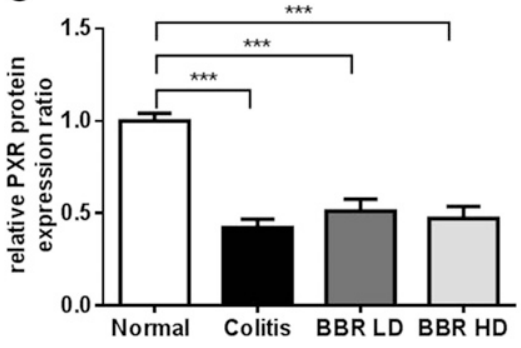

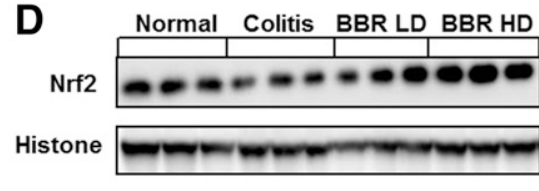

E

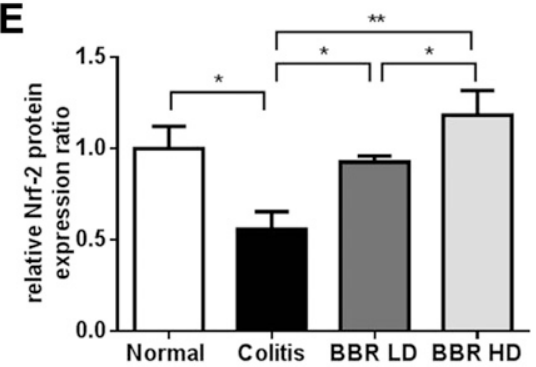

Fig. 4. Berberine increased P-gp and Nrf2 expression in DSS-induced colitis rats. (A and D) Representative Western blots and (B, C, and E) group data depicting P-gp, PXR, and nuclear Nrf2 expression in colon tissues from normal rats, colitis rats, and colitis rats treated with a low dose (LD, $10 \mathrm{mg} / \mathrm{kg}$ ) or high dose (HD, $40 \mathrm{mg} / \mathrm{kg}$ ) of berberine. Data represent the mean \pm S.E. M. ( $n=8$ rats per group). ${ }^{*} P<0.05 ; * * P<0.01 ; * * * P<$ 0.001 . Histone H3 was used as a loading control for nuclear extracts, and GAPDH was used as a loading control for cytoplasmic extracts.

symptoms in rats after berberine administration. The present study not only confirmed the beneficial effects of berberine on DSS-induced colitis in a rat model but also characterized the Nrf2-mediated mechanisms of P-gp upregulation through which berberine improved DSS-induced colitis.

Decreased P-gp expression and activity are implicated in IBD pathogenesis (Englund et al., 2007), and MDR1 has been shown to be a target gene for IBD therapy (Banner et al., 2004; Sehirli et al., 2015; Jing et al., 2016). Previous studies of an mdr1a-deficient colitis model demonstrated that $m d r 1 a^{-1-}$ mice spontaneously developed colonic inflammation that was histologically similar to that observed in human IBD (Panwala et al., 1998; Banner et al., 2004). Additionally, increased P-gp expression and/or function exhibited beneficial effects on colitis symptoms (Saksena et al., 2011; Jing et al., 2016). In agreement with earlier studies using different models, including DSS-induced mouse/rat colitis and trinitro-benzene-sulfonic acid-induced rat colitis (Iizasa et al., 2003; Sehirli et al., 2015; Jing et al., 2016), we observed decreased levels of $m d r 1 a$ mRNA and P-gp protein in the colonic tissues of the colitis rat model in the present study. Berberine treatment abrogated P-gp downregulation at both the mRNA and protein levels in DSS-induced colitis rats (Figs. 3 and 4), indicating that improvements in the intestinal barrier (via P-gp upregulation) contributed to the observed therapeutic effects of berberine on colitis. Berberine-induced P-gp upregulation was also reported in a previous study, in which berberine treatment of 24-hour upregulated P-gp expression and activity in various digestive track cancer cell lines (Lin et al., 1999b). Furthermore, berberine has been reported to upregulate $\mathrm{P}$-gp expression and activity in murine and human hepatoma cells (Lin et al., 1999a). One in vivo study demonstrated that berberine increased the bioavailability of digoxin, a P-gp substrate, through inhibition of intestinal P-gp (Qiu et al., 2009); however, a biphasic effect of berberine on P-gp ATPase activity was reported in the rat jejunal membrane (Najar et al., 2010). The differences seen in the in vivo and in vitro results may be due to differences in cell strains, dosages, substrates, and durations of exposure. Our findings from this study showing the berberine-mediated upregulation of P-gp expression are in agreement with the findings of most previous studies (Lin et al., 1999a; Qiu et al., 2009; Najar et al., 2010).

The transcriptional regulation of the $M D R 1$ gene is highly complex. The nuclear receptor PXR and the transcription factor Nrf2 play important roles in elevating MDR1 gene transcription and ultimately increasing P-gp function (Chen et al., 2012; Jeddi et al., 2018). Additionally, dysregulation of PXR/Nrf2 activity in the intestine is thought to contribute to colitis pathophysiology (Khor et al., 2006; Yang et al., 2017). In the present study of DSS-induced colitis rats, PXR and Nrf2 expression were decreased in the colon, in accordance with previous studies (Hu et al., 2014; Yang et al., 2017); however, in the berberine-treated group, only the decrease in Nrf2 expression was reversed by berberine, with no effect observed on PXR expression (Fig. 4). Although Yu et al. (2011) showed that berberine was an efficacious PXR agonist, their research was based on an in vitro experiment using HepG2 cells.

As a further mechanistic study, P-gp alteration in Caco-2 cells was investigated in response to berberine treatment. Because Caco-2 cells highly express P-gp, this cell line is used extensively for studying $\mathrm{P}$-gp regulatory mechanisms in physiologic and pathologic processes, such as colitis (Saksena et al., 2011, 2013; Jing et al., 2016). Using this in vitro model, we observed that berberine treatment upregulated P-gp expression and function (Fig. 5), a finding in accordance with previous in vitro studies (Shan et al., 2013). The role of Nrf2 in $\mathrm{P}$-gp expression has been investigated in many in vitro and 

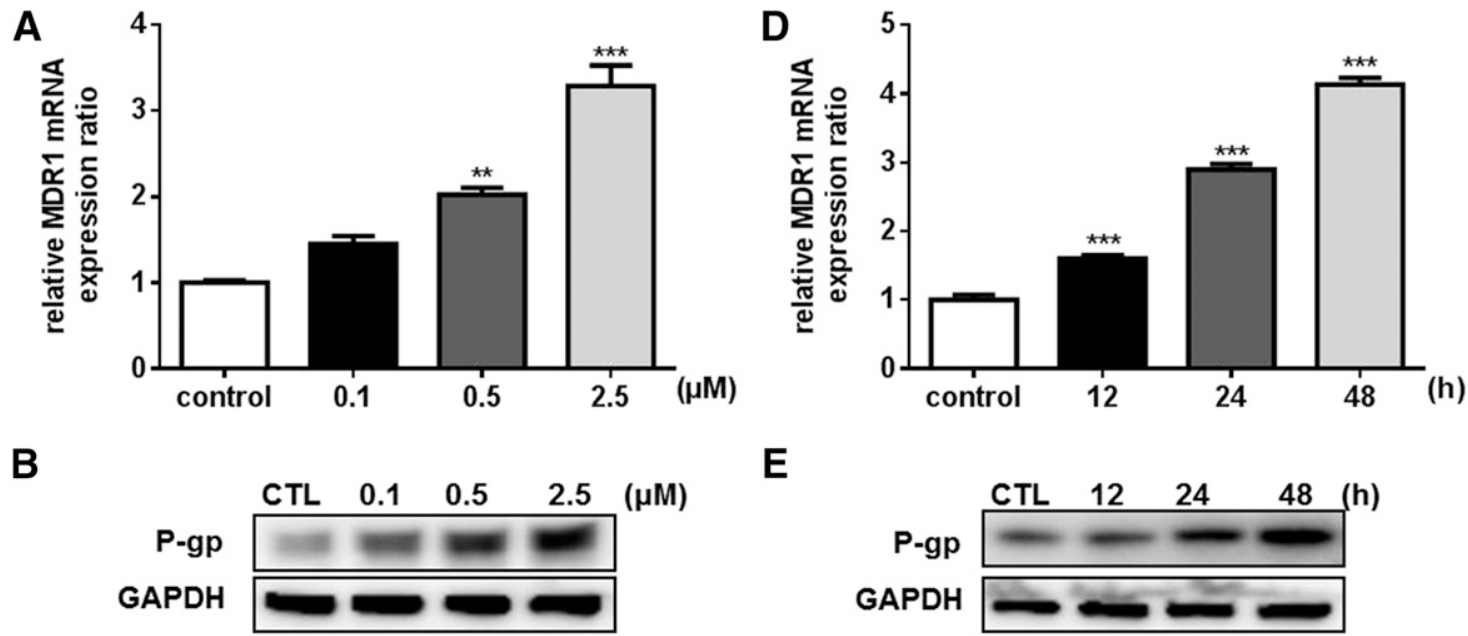

E
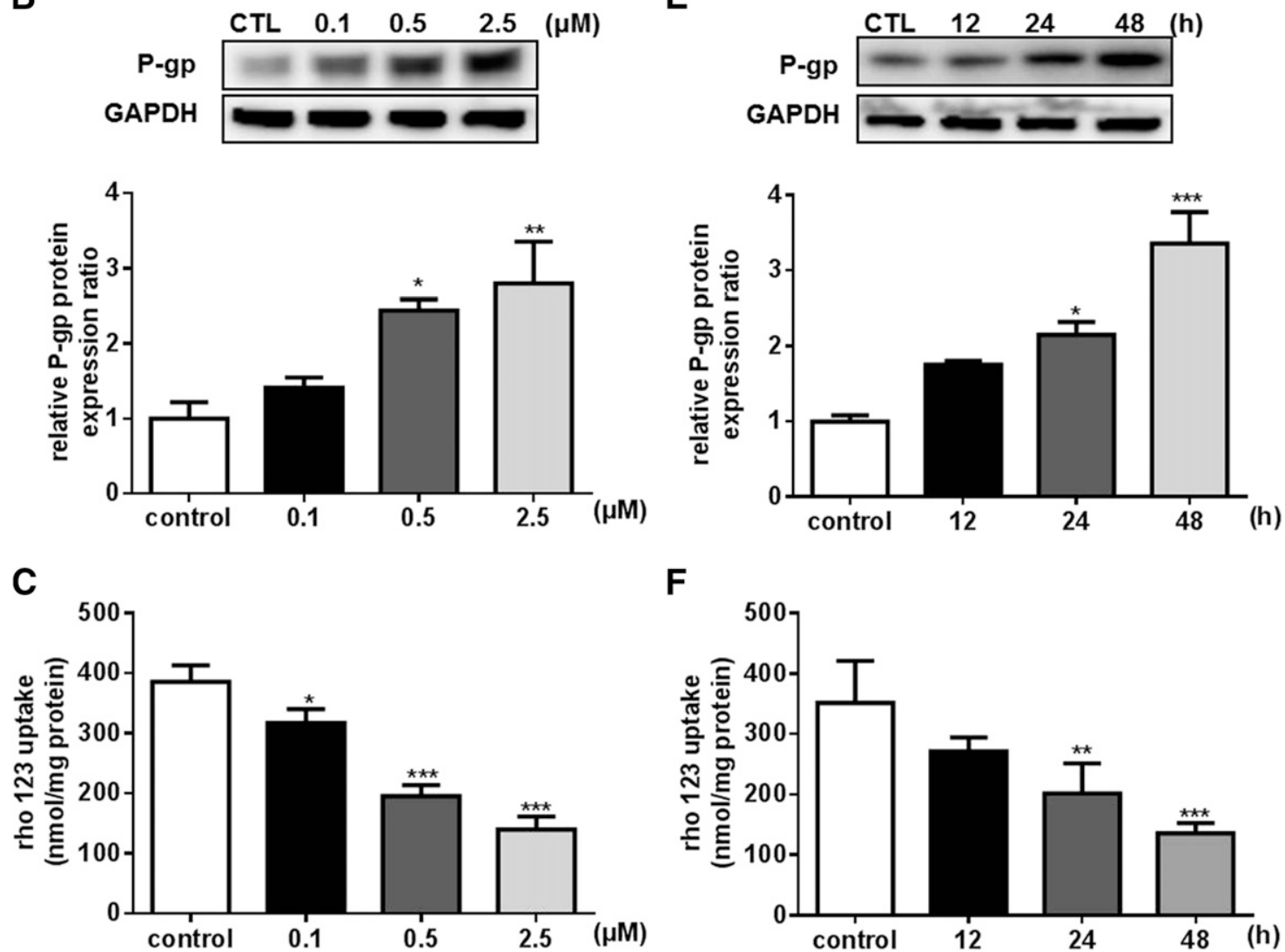

Fig. 5. P-gp was upregulated by berberine treatment in a dose- and time-dependent manner in Caco-2 cells. (A and D) MDR1 gene expression, (B and E) P-gp protein expression, and (C and F) P-gp function were induced by berberine treatment. qRT-PCR and Western blotting were performed to measure mRNA and protein levels, respectively. Cellular uptake of the P-gp substrate rho123 was used to measure P-gp function. Data represent the mean \pm S.E. M. of six samples from two independent experiments. ${ }^{*} P<0.05 ;{ }^{*} P<0.01$; ${ }^{* * *} P<0.001$ vs. control. GAPDH was used as a loading control for cytoplasmic extracts.

in vivo models (Wang et al., 2014; Jeong et al., 2015). Treatment with the Nrf2 inducer SFN upregulates P-gp protein levels in rat brains (Wang et al., 2014), and the activation of Nrf2 (through Keap1 knockdown) increases P-gp expression in human renal tubular cells (Jeong et al., 2015). To investigate whether berberine upregulates P-gp through Nrf2 activation, we used Caco-2 cells to perform an Nrf2 gene silencing experiment. Our data showed that the induction of MDR1 by berberine was abrogated by $N r f 2$ silencing (Fig. 6). Additionally, luciferase reporter assay and Western blotting results showed that berberine treatment increased $N r f 2$ reporter gene activity and upregulated the nuclear translocation of Nrf2 in Caco-2 cells (Fig. 6), suggesting that berberine is a strong Nrf2 activator. Previous studies also demonstrated the ability of berberine to activate Nrf2 nuclear translocation and promote its protective effects in different disease models (Zhang et al., 2016; Dinesh and Rasool, 2017; Mahmoud et al., 2017). These results suggest that the P-gp induction by berberine is achieved through activation of Nrf2.

In conclusion, our data demonstrated that P-gp expression was attenuated in DSS-induced colitis rat colons. Moreover, berberine treatment markedly alleviated the inflammatory processes involved in DSS-induced colitis and improved P-gp-mediated barrier function. In vivo and in vitro results demonstrated that the induction of P-gp expression and activity by berberine may occur via activation of the Nrf2mediated signaling pathway. The results of the current study suggest that the therapeutic effects of berberine on colitis are potentially due to the Nrf2-mediated upregulation of P-gp, 
A
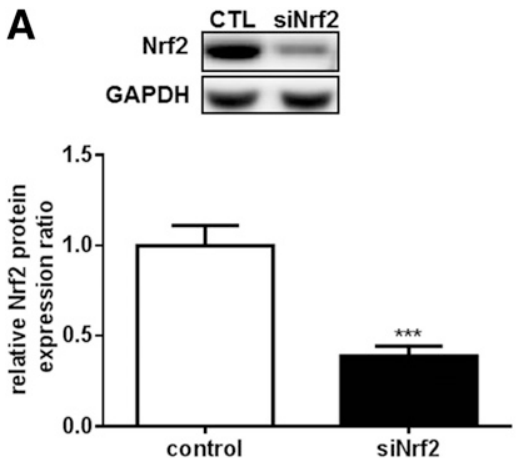

B
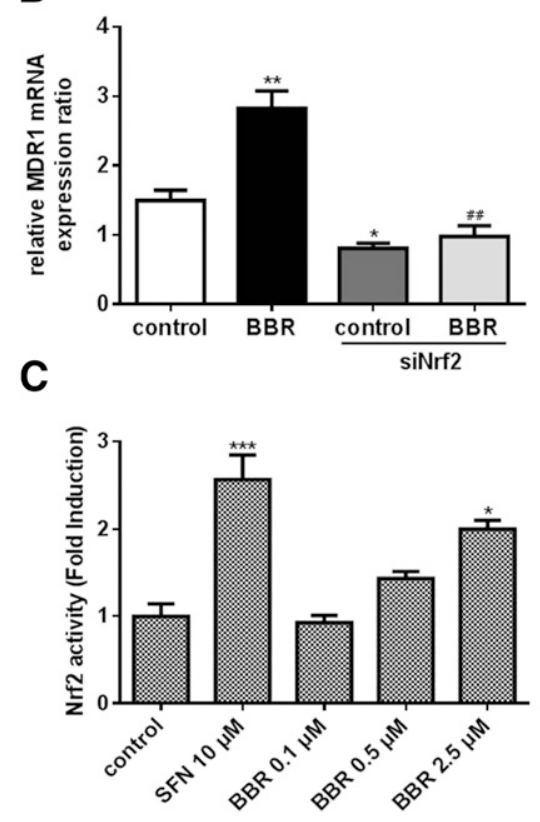

D

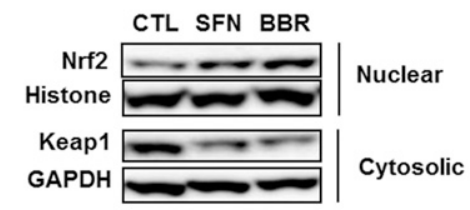

E

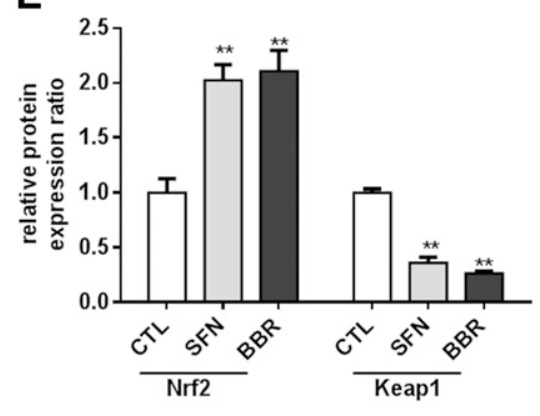

Fig. 6. Berberine upregulated the Nrf2/P-gp pathway in vitro. (A) Nrf2 silencing efficiency was measured by Western blotting, and GAPDH was used as a loading control. Data represent the mean \pm S.E.M. of six samples from two independent experiments. $* * * P<$ 0.001 vs. control. (B) Nrf2 knockdown abrogated P-gp induction by berberine in Caco-2 cells. qRT-PCR analysis was performed to measure mRNA expression, and data represent the mean \pm S.E.M. of six samples from two independent experiments. $* P<0.05$; $* * P<0.01 \mathrm{vs.}$ vehicle; ${ }^{\# \#} P<0.01$ vs. berberine alone. (C) Berberine treatment increased Nrf2 reporter gene activity. Caco-2 cells were transiently transfected with the ARE-luciferase plasmid and CMV Renilla luciferase plasmid, followed by treatment with different concentrations of berberine $(0.1,0.5$, or $2.5 \mu \mathrm{M})$ or the positive agonist SFN $(10 \mu \mathrm{M})$ for 24 hours. ${ }^{*} P<0.05 ; * * * P<0.001$ vs. control. (D and E) Representative Western blots and group data depicting nuclear translocation of Nrf2 and protein abundances of its repressor Keap1 in Caco-2 cells treated with berberine $(2.5 \mu \mathrm{M})$ or SFN $(10 \mu \mathrm{M})$ for 24 hours. Data represent the mean \pm S.E.M. of six samples from two independent experiments. ${ }^{* *} P<0.01$ vs. control.

thereby highlighting the potential of P-gp and/or Nrf2 as new targets for IBD therapy.

\section{Authorship Contributions}

Participated in research design: Jing, Fu, Wang.

Conducted experiments: Jing, Zhang, Guo, Chen.

Performed data analysis: Jing, Wu.

Wrote or contributed to the writing of the manuscript: Jing, Safarpour, Fu.

\section{References}

Aleksunes LM and Klaassen CD (2012) Coordinated regulation of hepatic phase I and II drug-metabolizing genes and transporters using AhR-, CAR-, PXR-, PPAR $\alpha$-, and Nrf2-null mice. Drug Metab Dispos 40:1366-1379.

Antonioli L, Fornai M, Colucci R, Ghisu N, Da Settimo F, Natale G, Kastsiuchenka O, Duranti E, Virdis A, Vassalle C, et al. (2007) Inhibition of adenosine deaminase attenuates inflammation in experimental colitis. J Pharmacol Exp Ther 322: $435-442$.

Ashrafi F, Kowsari F, Darakhshandeh A, and Adibi P (2014) Composite lymphoma in a patient with ulcerative colitis: a case report. Int $J$ Hematol Oncol Stem Cell Res 8: $45-48$.

Banner KH, Cattaneo C, Le Net JL, Popovic A, Collins D, and Gale JD (2004) Macroscopic, microscopic and biochemical characterisation of spontaneous colitis in a transgenic mouse, deficient in the multiple drug resistance 1a gene. Br J Pharmacol 143:590-598.

Blokzijl H, Vander Borght S, Bok LI, Libbrecht L, Geuken M, van den Heuvel FA, Dijkstra G, Roskams TA, Moshage H, Jansen PL, et al. (2007) Decreased $\mathrm{P}$-glycoprotein (P-gp/MDR1) expression in inflamed human intestinal epithelium is independent of PXR protein levels. Inflamm Bowel Dis 13:710-720.

Brinar M, Cukovic-Cavka S, Bozina N, Ravic KG, Markos P, Ladic A, Cota M, Krznaric Z, and Vucelic B (2013) MDR1 polymorphisms are associated with inflammatory bowel disease in a cohort of Croatian IBD patients. BMC Gastroenterol 13:57.
Chen Y, Tang Y, Guo C, Wang J, Boral D, and Nie D (2012) Nuclear receptors in the multidrug resistance through the regulation of drug-metabolizing enzymes and drug transporters. Biochem Pharmacol 83:1112-1126.

Cheng J, Shah YM, Ma X, Pang X, Tanaka T, Kodama T, Krausz KW, and Gonzalez FJ (2010) Therapeutic role of rifaximin in inflammatory bowel disease: clinical implication of human pregnane X receptor activation. J Pharmacol Exp Ther 335: $32-41$.

Chu XY, Strauss JR, Mariano MA, Li J, Newton DJ, Cai X, Wang RW, Yabut J, Hartley DP, Evans DC, et al. (2006) Characterization of mice lacking the multidrug resistance protein MRP2 (ABCC2). J Pharmacol Exp Ther 317: 579-589.

Dinesh P and Rasool M (2017) Berberine, an isoquinoline alkaloid suppresses TXNIP mediated NLRP3 inflammasome activation in MSU crystal stimulated RAW 264.7 macrophages through the upregulation of Nrf2 transcription factor and alleviates MSU crystal induced inflammation in rats. Int Immunopharmacol 44:26-37.

Englund G, Jacobson A, Rorsman F, Artursson P, Kindmark A, and Rönnblom A (2007) Efflux transporters in ulcerative colitis: decreased expression of BCRP (ABCG2) and Pgp (ABCB1). Inflamm Bowel Dis 13:291-297.

Gu L, Li N, Li Q, Zhang Q, Wang C, Zhu W, and Li J (2009) The effect of berberine in vitro on tight junctions in human Caco-2 intestinal epithelial cells. Fitoterapia 80:241-248.

Gutmann H, Hruz P, Zimmermann C, Straumann A, Terracciano L, Hammann F, Lehmann F, Beglinger C, and Drewe J (2008) Breast cancer resistance protein and P-glycoprotein expression in patients with newly diagnosed and therapy-refractory ulcerative colitis compared with healthy controls. Digestion 78:154-162.

Habtemariam S (2016) Berberine and inflammatory bowel disease: a concise review. Pharmacol Res 113 (Pt A):592-599.

Hong T, Yang Z, Lv CF, and Zhang Y (2012) Suppressive effect of berberine on experimental dextran sulfate sodium-induced colitis. Immunopharmacol Immunotoxicol 34:391-397.

Hu D, Wang Y, Chen Z, Ma Z, You Q, Zhang X, Zhou T, Xiao Y, Liang Q, Tan H, et al. (2014) Artemisinin protects against dextran sulfate-sodium-induced inflammatory bowel disease, which is associated with activation of the pregnane X receptor. Eur $J$ Pharmacol 738:273-284.

Huang Y, Hu N, Gao X, Yan Z, Li S, Jing W, and Yan R (2015) Alterations of testosterone metabolism in microsomes from rats with experimental colitis induced by dextran sulfate sodium. Chem Biol Interact 232:38-48. 
Huls M, Russel FG, and Masereeuw R (2009) The role of ATP binding cassette transporters in tissue defense and organ regeneration. J Pharmacol Exp Ther 328 $3-9$.

Iizasa H, Genda N, Kitano T, Tomita M, Nishihara K, Hayashi M, Nakamura K, Kobayashi S, and Nakashima E (2003) Altered expression and function of $\mathrm{P}$-glycoprotein in dextran sodium sulfate-induced colitis in mice. J Pharm Sci 92: 569-576.

Jeddi F, Soozangar N, Sadeghi MR, Somi MH, Shirmohamadi M, Eftekhar-Sadat AT, and Samadi N (2018) Nrf2 overexpression is associated with P-glycoprotein upregulation in gastric cancer. Biomed Pharmacother 97:286-292.

Jeong HS, Ryoo IG, and Kwak MK (2015) Regulation of the expression of renal drug transporters in KEAP1-knockdown human tubular cells. Toxicol In Vitro 29 884-892.

Jing W, Vaziri ND, Nunes A, Suematsu Y, Farzaneh T, Khazaeli M, and Moradi H (2017) LCZ696 (Sacubitril/valsartan) ameliorates oxidative stress, inflammation, fibrosis and improves renal function beyond angiotensin receptor blockade in CKD. Am J Transl Res 9:5473-5484.

Jing WH, Gao XJ, Han BL, Wei B, Hu N, Li S, Yan R, and Wang YT (2016) Mori cortex regulates $\mathrm{P}$-glycoprotein in Caco-2 cells and colons from rats with experimental colitis via direct and gut bacteria-mediated mechanisms. RSC Adv 7: $2594-2605$

Johnstone RW, Ruefli AA, and Smyth MJ (2000) Multiple physiological functions for multidrug transporter P-glycoprotein? Trends Biochem Sci 25:1-6.

Khor TO, Huang MT, Kwon KH, Chan JY, Reddy BS, and Kong AN (2006) Nrf2deficient mice have an increased susceptibility to dextran sulfate sodium-induced colitis. Cancer Res 66:11580-11584

Lee IA, Hyun YJ, and Kim DH (2010) Berberine ameliorates TNBS-induced colitis by inhibiting lipid peroxidation, enterobacterial growth and NF-кB activation. Eur $J$ Pharmacol 648:162-170.

Li YH, Xiao HT, Hu DD, Fatima S, Lin CY, Mu HX, Lee NP, and Bian ZX (2016) Berberine ameliorates chronic relapsing dextran sulfate sodium-induced colitis in C57BL/6 mice by suppressing Th17 responses. Pharmacol Res 110:227-239.

Lin HL, Liu TY, Lui WY, and Chi CW (1999a) Up-regulation of multidrug resistance transporter expression by berberine in human and murine hepatoma cells. Cancer 85:1937-1942.

Lin HL, Liu TY, Wu CW, and Chi CW (1999b) Berberine modulates expression of mdr1 gene product and the responses of digestive track cancer cells to paclitaxel. $\mathrm{Br} J$ Cancer 81:416-422.

Liu Y, Liu X, Hua W, Wei Q, Fang X, Zhao Z, Ge C, Liu C, Chen C, Tao Y, et al. (2018) Berberine inhibits macrophage M1 polarization via AKT1/SOCS1/NF-кB signaling pathway to protect against DSS-induced colitis. Int Immunopharmacol $\mathbf{5 7}$ 121-131.

Maeda S, Hsu LC, Liu H, Bankston LA, Iimura M, Kagnoff MF, Eckmann L, and Karin M (2005) Nod2 mutation in Crohn's disease potentiates NF-kappaB activity and IL-1beta processing. Science 307:734-738.

Mahmoud AM, Hozayen WG, and Ramadan SM (2017) Berberine ameliorates methotrexate-induced liver injury by activating Nrf2/HO-1 pathway and PPAR $\gamma$, and suppressing oxidative stress and apoptosis in rats. Biomed Pharmacother $\mathbf{9 4}$ $280-291$.

Martin JC, Bériou G, and Josien R (2016) Dextran sulfate sodium (DSS)-induced acute colitis in the rat. Methods Mol Biol 1371:197-203.

Metzler KD, Fuchs TA, Nauseef WM, Reumaux D, Roesler J, Schulze I, Wahn V, Papayannopoulos V, and Zychlinsky A (2011) Myeloperoxidase is required for neutrophil extracellular trap formation: implications for innate immunity. Blood 117:953-959.

Miner-Williams WM and Moughan PJ (2016) Intestinal barrier dysfunction: implications for chronic inflammatory conditions of the bowel. Nutr Res Rev 29:40-59.

Najar IA, Sachin BS, Sharma SC, Satti NK, Suri KA, and Johri RK (2010) Modulation of P-glycoprotein ATPase activity by some phytoconstituents. Phytother Res 24:454-458.

Panwala CM, Jones JC, and Viney JL (1998) A novel model of inflammatory bowel disease: mice deficient for the multiple drug resistance gene, mdr1a, spontaneously develop colitis. J Immunol 161:5733-5744

Peterson CG, Lampinen M, Hansson T, Lidén M, Hällgren R, and Carlson M (2016) Evaluation of biomarkers for ulcerative colitis comparing two sampling methods: fecal markers reflect colorectal inflammation both macroscopically and on a cellular level. Scand J Clin Lab Invest 76:393-401.
Qiu W, Jiang XH, Liu CX, Ju Y, and Jin JX (2009) Effect of berberine on the pharmacokinetics of substrates of CYP3A and P-gp. Phytother Res 23:1553-1558.

Saksena S, Goyal S, Raheja G, Singh V, Akhtar M, Nazir TM, Alrefai WA, Gill RK and Dudeja PK (2011) Upregulation of P-glycoprotein by probiotics in intestinal epithelial cells and in the dextran sulfate sodium model of colitis in mice. Am J Physiol Gastrointest Liver Physiol 300:G1115-G1123.

Saksena S, Priyamvada S, Kumar A, Akhtar M, Soni V, Anbazhagan AN, Alakkam A Alrefai WA, Dudeja PK, and Gill RK (2013) Keratinocyte growth factor-2 stimulates P-glycoprotein expression and function in intestinal epithelial cells. Am J Physiol Gastrointest Liver Physiol 304:G615-G622.

Sehirli AO, Cetinel S, Ozkan N, Selman S, Tetik S, Yuksel M, and Dulger FG (2015) St. John's wort may ameliorate 2,4,6-trinitrobenzenesulfonic acid colitis off rats through the induction of pregnane X receptors and/or P-glycoproteins. J Physiol Pharmacol 66:203-214.

Shan YQ, Ren G, Wang YX, Pang J, Zhao ZY, Yao J, You XF, Si SY, Song DQ, Kong WJ, et al. (2013) Berberine analogue IMB-Y53 improves glucose-lowering efficacy by averting cellular efflux especially P-glycoprotein efflux. Metabolism 62:446-456. Sharom FJ (2011) The P-glycoprotein multidrug transporter. Essays Biochem 50: 161-178.

Shitan N, Tanaka M, Terai K, Ueda K, and Yazaki K (2007) Human MDR1 and MRP1 recognize berberine as their transport substrate. Biosci Biotechnol Biochem 71:242-245.

Tan S, Yu W, Lin Z, Chen Q, Shi J, Dong Y, Duan K, Bai X, Xu L, Yu Z, et al. (2015) Berberine ameliorates intestinal mucosal barrier damage induced by peritoneal air exposure. Biol Pharm Bull 38:122-126.

Tili E, Michaille JJ, Piurowski V, Rigot B, and Croce CM (2017) MicroRNAs in intestinal barrier function, inflammatory bowel disease and related cancers-their effects and therapeutic potentials. Curr Opin Pharmacol 37:142-150.

Wang X, Campos CR, Peart JC, Smith LK, Boni JL, Cannon RE, and Miller DS (2014) Nrf2 upregulates ATP binding cassette transporter expression and activity at the blood-brain and blood-spinal cord barriers. J Neurosci 34:8585-8593.

Yan F, Wang L, Shi Y, Cao H, Liu L, Washington MK, Chaturvedi R, Israel DA, Cao $\mathrm{H}$, Wang B, et al. (2012) Berberine promotes recovery of colitis and inhibits inflammatory responses in colonic macrophages and epithelial cells in DSS-treated mice. Am J Physiol Gastrointest Liver Physiol 302:G504-G514.

Yang N, Xia Z, Shao N, Li B, Xue L, Peng Y, Zhi F, and Yang Y (2017) Carnosic acid prevents dextran sulfate sodium-induced acute colitis associated with the regulation of the Keap1/Nrf2 pathway. Sci Rep 7:11036.

Yu C, Chai X, Yu L, Chen S, and Zeng S (2011) Identification of novel pregnane X receptor activators from traditional Chinese medicines. $J$ Ethnopharmacol 136 : 137-143

Zhang J, Ding L, Wang B, Ren G, Sun A, Deng C, Wei X, Mani S, Wang Z, and Dou W (2015a) Notoginsenoside R1 attenuates experimental inflammatory bowel disease via pregnane X receptor activation. J Pharmacol Exp Ther 352:315-324.

Zhang X, Liang D, Lian X, Jiang Y, He H, Liang W, Zhao Y, and Chi ZH (2016) Berberine activates Nrf2 nuclear translocation and inhibits apoptosis induced by high glucose in renal tubular epithelial cells through a phosphatidylinositol 3-kinase/Akt-dependent mechanism. Apoptosis 21:721-736.

Zhang X, Qiu F, Jiang J, Gao C, and Tan Y (2011) Intestinal absorption mechanisms of berberine, palmatine, jateorhizine, and coptisine: involvement of P-glycoprotein Xenobiotica 41:290-296.

Zhang X, Wang Y, Ma Z, Liang Q, Tang X, Hu D, Tan H, Xiao C, and Gao Y (2015b) Tanshinone IIA ameliorates dextran sulfate sodium-induced inflammatory bowel disease via the pregnane X receptor. Drug Des Devel Ther 9:6343-6362.

Zhao JJ, Wang D, Yao H, Sun DW, and Li HY (2015) CTLA-4 and MDR1 polymorphisms increase the risk for ulcerative colitis: a meta-analysis. World J Gastroenterol 21:10025-10040.

Zhou H and Mineshita S (2000) The effect of berberine chloride on experimental colitis in rats in vivo and in vitro. $J$ Pharmacol Exp Ther 294:822-829.

Address correspondence to: Qiang Fu, School of Pharmacy, Xi'an Jiaotong University, Xi'an, Shaanxi, China. E-mail: fuqiang@mail.xjtu.edu.cn; or Wanghui Jing, School of Pharmacy, Xi'an Jiaotong University, Xi'an, Shaanxi, China. E-mail: jingwanghui1987@163.com 